\section{Vorschläge für eine rationale und rationelle Diagnostik des Hustens ${ }^{1}$}

Zusammenfassung: Husten ist das häufigste Symptom pneumologischer Erkrankungen. Eine zweckmäßige und kostengünstige Stufendiagnostik des Hustens ist daher unerläßlich. Beispielsweise sollte die Bagatellerkrankung akute Bronchitis möglichst wenig diagnostischen Aufwand beanspruchen. Die meisten Patienten mit akutem oder chronischem (über acht Wochen anhaltendem) Husten können bereits mit Hilfe der Anamnese, der körperlichen Untersuchung, Röntgenaufnahme des Thorax und der Lungenfunktionsprüfung abgeklärt werden. Diese Verfahren (Basisdiagnostik) bilden die erste und zweite Stufe des vorgeschlagenen diagnostischen Stufenschemas. Besonders die Abklärung des chronischen Hustens nicht evidenter Ursache (chronisch persistierender Husten, $\mathrm{CPH}$ ) bedarf hingegen einer viel subtileren Diagnostik (unspezifische inhalative Provokationstestung, 24-Stunden-pH-Metrie, Bronchoskopie, CT des Thorax und der Nasennebenhöhlen, hals-nasen-ohrenärztliche oder neurologische Untersuchung). Trotzdem bleibt die Ursache des Hustens bei bis zu $20 \%$ solcher CPH-Patienten ungeklärt. Es wird je ein Flussdiagramm zur Diagnostik des akuten und des chronischen Hustens (Abb. 1 und 2) vorgestellt. Es handelt sich hierbei um einen hierarchisch angeordneten Algorithmus der diagnostischen Methoden. Er berücksichtigt die Häufigkeit der Erkrankungen, den positiven prädiktiven Wert, die Invasivität und die Kosten, soweit verfügbar. Die häufigsten Verletzungen des Prinzips einer abgestuften Diagnostik werden kritisch abgehandelt.

Proposals for a Diagnostic Algorithm for Acute and Chronic Cough: Cough is probably the most frequent symptom in chest diseases. Hence, a rational and economical diagnostic procedure is essential to prevent unnecessary costs to the health services, i.e. acute bronchitis, a self-limiting disease, which is the most frequent cause for cough should not involve extensive per case costs. History, physical examination, chest $X$-ray and lung function testing - which constitute both the first and second, i.e. the basic level of a stepwise approach - allows to diagnose causes in most patients with cough. Without evidence of the cause after completing this basic diagnostic procedure patients with acute cough may require blood gases analysis, electrocardiography, echocardiography, lung perfusion study, spiral CT angiography, bronchoscopy or laboratory examinations for diagnosis of pulmonary embolism, aspiration or (seldom) pleuritis sicca. Chronic persistent cough (CPC) is diagnosed if the basic standard approach to chronic cough fails to lead

Pneumologie 2000; 54: 110-115

(C) Georg Thieme Verlag Stuttgart · New York ISSN 0934-8387

\section{P. Kardos}

Gemeinschaftspraxis und pneuologisch-allergologisches Zentrum Maingau Krankenhaus T. Gebhardt, A. Iwantscheff, P. Kardos, Frankfurt am Main

to final diagnosis. Patients will then need further subtle diagnostic management, i.e. bronchial provocation testing, 24 hour $\mathrm{pH}$ probe, ENT- or neurological examination, high resolution $\mathrm{CT}$ of the thorax and bronchoscopy. We present two algorithms for the rational diagnostic approach to acute (figure 1) and chronic (figure 2) cough. Each algorithm considers spectrum and frequency of causes on the one hand, the positive predictive value, costs and patient discomfort due to the examination on the other. Nonetheless, despite extensive examination up to $20 \%$ of patients suffering from CPC the cause remains unclear [11]. Frequently, the capsaicin cough challenge test can reveal an idiopathic upregulation of the cough reflex as the hypothesised underlying condition. Psychogenic cough however, a rare condition in adults should not coincide with hypersensitivity of the cough reflex. Inconsistency and low reproducibility of results of the capsaicin challenge in patients with psychogenic cough preclude his routine clinical use. In conclusion, the very common acute bronchitis and the ACE inhibitor - induced cough do not require any other diagnostic procedure except patient history and physical examination. A simple basic diagnostic approach will usually allow to evaluate acute and chronic cough. In the remaining cases the proposed algorithm should be used for best results and to prevent excessive costs.

\section{Einleitung}

Husten ist ein gemeinsames Symptom beinahe aller pneumologischen Erkrankungen, auch die sehr häufige akute Bronchitis - isoliert oder in Rahmen grippaler Infekte auftretend geht mit Husten einher. Somit kommt Husten außerordentlich häufig vor, in einer repräsentativen Befragung von 1502 über 60-jährigen Personen war Husten - ebenso wie gastrointestinale Erkrankungen die am häufigsten geäußerte Beschwerde [17]; in den USA war er der dritthäufigste Grund, einen niedergelassenen Internisten aufzusuchen [1]. Der im Rahmen des Symptoms Husten doch kleine Anteil von Patienten mit chronischem Husten nicht evidenter Ursache (chronisch persistierender Husten, $\mathrm{CPH}$ ) entsprach immerhin $5,7 \%$ aller neu in unsere Gemeinschaftspraxis für Allergologie und Pneumologie überwiesenen Patienten [11].

Mit diesen Zahlen sollen die Inanspruchnahme des Gesundheitssystems und die wirtschaftliche Bedeutung einer ratio-

1 Teilweise vorgetragen auf dem 40. Kongress der Deutschen Gesellschaft für Pneumologie 25. Kongress der Österreichischen Gesellschaft für Lungenerkrankungen und Tuberkulose in Bad Reichenhall/ Salzburg am 17. März 1999 
nalen (schnell zum Ziele führenden) und rationellen (sparsamen) Diagnostik des Hustens hervorgehoben werden.

Ein „Zuviel“ an Diagnostik:

- (Computertomographie, Bronchoskopie bei einer banalen, protrahiert verlaufenden akuten Bronchitis z.B. nach Mykoplasmeninfekt),

ebenso wie „zu wenig“

- (die Bagatellisierung des „Raucherhustens“, welcher Symptom einer ansteckenden Tuberkulose oder eines Bronchialkarzinoms sein kann)

belasten die knapp gewordenen finanziellen Ressourcen mit direkten und indirekten Folgekosten in kaum berechenbarer Höhe. Darüber hinaus vermehren sie das Leid der Patienten.

Dieser Artikel soll ein diagnostisches Stufenschema in die Hand der Allgemeinärzte, Internisten, Pädiater und Pneumologen geben, die in der überwiegenden Mehrzahl der Fälle den Husten ambulant in der Praxis oder gar beim Hausbesuch abklären müssen.

Da Husten das gemeinsame Symptom eines breiten Spektrums von Erkrankungen mit sehr unterschiedlichem Therapieansatz und Prognose ist - erscheint eine Klassifizierung des Hustens anhand eines anamnestisch leicht zu erhebenden Merkmals: akut und chronisch - als erster Schritt angezeigt, wenngleich Überschneidungen zwischen den Gruppen nicht ganz zu vermeiden sind (Tab. 1).

Tab. 1 Wichtige klinische Ursachen des Hustens und ihre Klassifizierung

\begin{tabular}{lll}
\hline akut $(<8$ Wochen) & chronisch $(>8$ Wochen) & Diagnostik \\
\hline $\begin{array}{l}\text { akute Bronchitis } \\
\text { inhalative Intoxikation }\end{array}$ & $\begin{array}{l}\text { Asthma } \\
\text { Asthma }\end{array}$ & $\begin{array}{l}\text { Stufe } 1 \\
\text { COPD }\end{array}$ \\
$\begin{array}{l}\text { Pneumothorax } \\
\text { Pneumonie }\end{array}$ & Bronchialkarzinom & \\
Aspiration & Tbc & \\
Linksherzinsuffizienz & Fibrose & \\
& Aspiration & \\
& Linksherzinsuffizienz & \\
& CPH chronisch & Stufe 1+2+3 \\
persistierender Husten & \\
Lungenembolie & & Spezial- \\
Pleuritis & & diagnostik \\
\hline
\end{tabular}

\section{Akuter und chronischer Husten}

Der Spontanverlauf des Hustens bei der akuten Bronchitis beträgt bis zum völligen Abklingen durchschnittlich vier Wochen [2]. Adenoviren, Mykoplasmen verursachen in der Regel sechs bis acht Wochen anhaltenden Husten, nach Infektion mit B. pertussis husten die Patienten (vor allem Kinder, nur ausnahmsweise Erwachsene) noch viel länger. Wir empfehlen deshalb - falls eine typische Anamnese passend $\mathrm{zu}$ einer akuten Bronchitis vorliegt und anamnestisch keine verdächtigen Symptome (Hämoptoe, extrem starker Raucher,
Tab. 2 Stufendiagnostik beim Husten

Stufe 1 (alle Patienten)
Anamnese
körperliche Untersuchung
Stufe 2 (Basisdiagnostik)
Röntgen Thoraxaufnahme
Lungenfunktionsprüfung
Stufe 3
unspezifischer inhalativer Provokationstest
hals-nasen-ohrenärztliche Untersuchung, ggf. Sonographie,
Röntgen, CT der Nebenhöhlen
Stufe 4
24 Stunden pH-Metrie
CT des Thorax
Bronchoskopie

Fieber, schwere Begleiterkrankungen) offenkundig werden, acht Wochen bis zum Beginn der Stufendiagnostik (Tab. 2) abzuwarten. Wir schlagen daher die willkürlich gezogene Grenze von acht Wochen seit Beginn der Beschwerden (nicht etwa ab Beginn der Therapie, die häufig viel später erfolgt!) als Grenze zwischen „akut“ und „chronisch“ vor.

Nach unserer Erfahrung mit 329 Patienten [11] ist dieser Ansatz praxisgerecht. Bei Abklärung des chronischen Hustens geht es nicht um Wochen, sondern um Monate oder Jahre: $42 \%$ unserer Patienten gaben eine Beschwerdedauer von mehr als einem Jahr an.

Die rationelle, bezahlbare Diagnostik erfordert sowohl ein stufenweises Vorgehen (Tab. 2) als auch in manchen Fällen die zügige, volle Ausschöpfung des diagnostischen Armamentariums. Falls kein richtungweisender anamnestischer Hinweis auf die Ursache des Hustens vorliegt, helfen statistische Häufigkeit der infrage kommenden Erkrankungen, Kosten und Invasivität der einzelnen Untersuchungsmethoden die rationale und rationelle Reihenfolge der diagnostischen Schritte festzulegen, wie sie für den akuten und chronischen Husten getrennt in Flußdiagrammen (Abb.1 u. 2) dargestellt werden. Obgleich ein kürzlich publizierter „Consensus Statement“ der American College of Chest Physicians [18] ähnliche Empfehlungen ausspricht, muß darauf hingewiesen werden, daß die Flußdiagramme keine im Sinne der „evidence based medicine“ prospektiv validierte Vorgehensweise darstellen. Vielmehr sollte hierdurch zunächst eine Diskussionsgrundlage für eine spätere solche Empfehlung geschaffen werden.

\section{Akuter Husten}

Tab. 1 zeigt die wichtigsten Erkrankungen, welche akuten Husten verursachen können. Die rechte Spalte der Tab. 1 ordnet die entsprechende Stufe des diagnostischen Schemas aus Tab. 2 den korrespondierenden Diagnosen zu.

Die akute Bronchitis besetzt dabei eine Sonderstellung: Sie zeichnet zahlenmäßig für die Mehrzahl der Patienten mit dem Symptom Husten verantwortlich. Für die Diagnose reicht jedoch die Anamnese und (zum Ausschluß anderer Ursachen) 
eine körperliche Untersuchung aus, in manchen Fällen lässt sich gar eine ätiologisch begründete (Verdachts-)Diagnose stellen:

- Akuter Husten im Rahmen einer Grippeepidemie wird auf einen grippalen Infekt (Myxovirus influenzae A oder B) zurückzuführen sein.

- Junge Männer neigen zu Mycoplasma pneumoniae oder Adenovirusinfektion, besonders wenn sie kaserniert sind.

- Allergiker neigen zu Rhinovirusinfekten, da durch Allergenexposition die Expression des Zytokins ICAM 1 stimuliert wird, ICAM 1 ist morphologisch identisch mit den Rhinovirusrezeptoren der Schleimhaut.

- Kinderkrankheiten wie Keuchhusten, Masern, Scharlach, das Pfeiffersche Drüsenfieber lösen oft eine akute Bronchitis aus.

- Akute inhalative Intoxikationen (Unfälle am Arbeitsplatz, Brände) verursachen meistens gleichzeitig Bronchitis, Konjunktivitis und Rhinitis.

Da die akute Bronchitis nicht nur eine banale, sondern auch spontan ausheilende Erkrankung ist, erfordert sie in der Regel weder eine radiologische noch mikrobiologische oder Labordiagnostik. Bei typischer Anamnese für einen banalen Infekt und ohne klinischen Hinweis auf eine schwere (Begleit-) Erkrankung kann bis zur Einleitung der Stufe 2 der Diagnostik (s. Tab. 2) die mögliche Verlaufszeit der akuten Bronchitis bis zu acht Wochen - abgewartet werden. Die Anamnese mit dem Beschwerdebeginn ist schriftlich festzuhalten, da der Patient sich später ggf. nicht mehr an den Beginn seiner banalen Beschwerden erinnern wird. Während der normalen Verlaufszeit sind keine diagnostischen Maßnahmen indiziert. Falls die Ursache des akuten Hustens mittels Anamnese und körperlicher Untersuchung nicht überzeugend zu klären ist (d.h. wenn es sich nicht um eine klassische akute - entweder isoliert, oder im Rahmen eines allgemeinen viralen Infektes auftretende - Bronchitis handelt, ist die Stufe 2 der Diagnostik - abweichend hiervon - sofort einzuleiten.

Auch eine akute eosinophile Bronchitis, oft mit bronchialer Hyperreagibilität bei inhalativer Typ-I-Allergie kann zu akutem Husten führen. Sie läßt sich durch die allergologische Anamnese und eine Allergie-Hauttestung diagnostizieren. Allerdings gibt es Fälle von eosinophiler Bronchitis auch ohne Allergien (infektbedingt, idiopathisch). Im Sputum finden sich eosinophile Granulozyten.

Für weitere Auslöser eines akuten Hustens gilt: bis auf wenige Ausnahmen führt bereits die relativ kostengünstige und breit verfügbare Basisdiagnostik (Stufe 2, Tab. 2) zu einer definitiven Diagnose, wie im mittleren Teil der Tab. 1 vorgestellt.

Tab. 3 Weiterführende Spezialdiagnostik beim Husten

High Resolution-CT

Spiral-CT mit Kontrastmittel

Perfusionsszintigraphie

mikrobiologische Diagnostik

Labordiagnostik

Allergiediagnostik

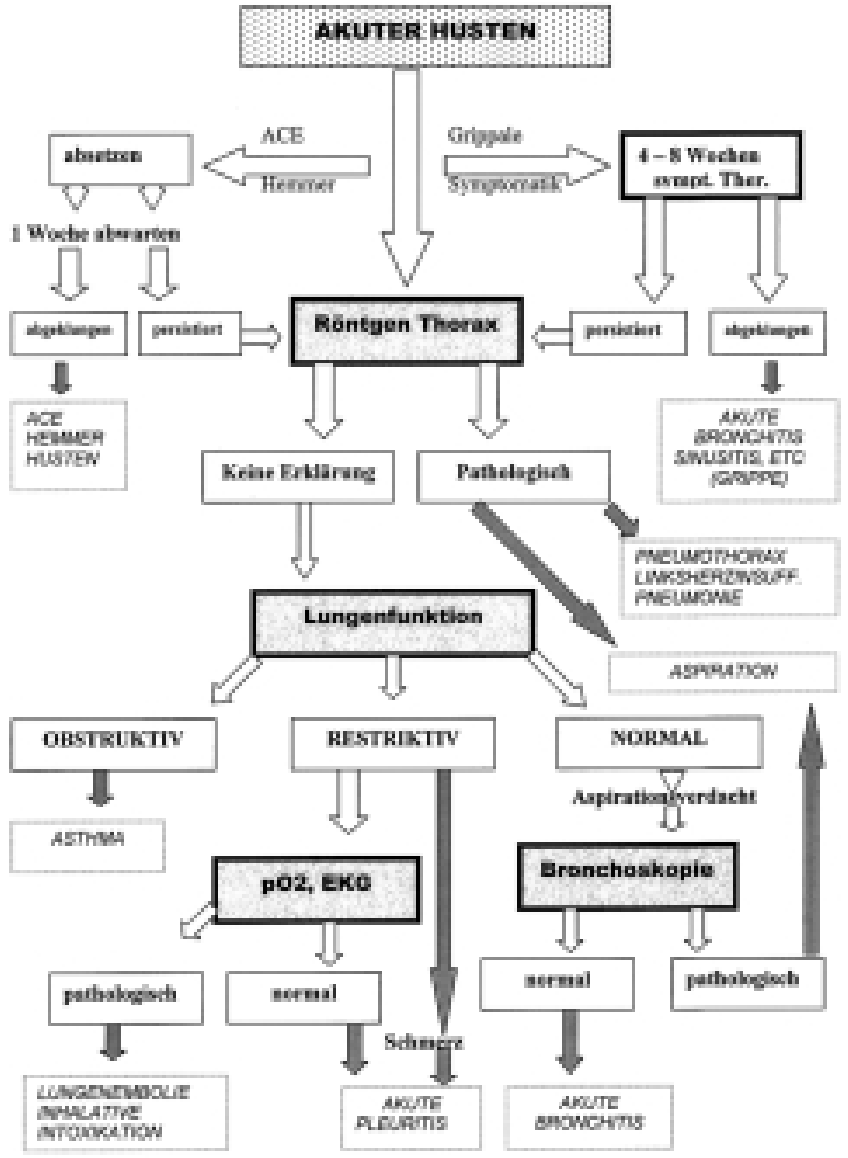

Abb. 1 Flussdiagramm akuter Husten.

Zur Diagnose von Lungenembolie oder Pleuritis sicca ist allerdings eine weiterführende Spezialdiagnostik (Tab.3) erforderlich. Wenn eine Verdachtsdiagnose der den Husten auslösenden Erkrankung bereits gestellt wurde, sind weitere gezielte diagnostische Maßnahmen erforderlich. Sie gehören dann nicht mehr sensu stricto zur Diagnostik des Hustens und werden hier nicht im Einzelnen aufgeführt. Betreffend die weitere Diagnostik sei auf die entsprechenden Empfehlungen der Fachgesellschaften hingewiesen.

Abb. 1 stellt ein Flußdiagramm für die rationale und rationelle Diagnostik des akuten Hustens dar. Das Flußdiagramm dient zur Abklärung unklarer Fälle. Bei einem konkreten Patienten mit begründeter Verdachtsdiagnose kann es evtl. rational und rationell sein, hiervon abweichend zu verfahren.

\section{Chronischer Husten}

Abb. 2 zeigt das Flußdiagramm für die rationale und rationelle Diagnostik des chronischen Hustens.

Wenn ein Patient sich mit chronischem, über acht Wochen anhaltendem Husten präsentiert, sollte die Basisdiagnostik (die ersten beiden Stufen in Tab. 2) sofort durchgeführt werden. Es sei denn, der Patient wird mit ACE-Hemmer (angiotensin converting enzyme Hemmer) behandelt. 


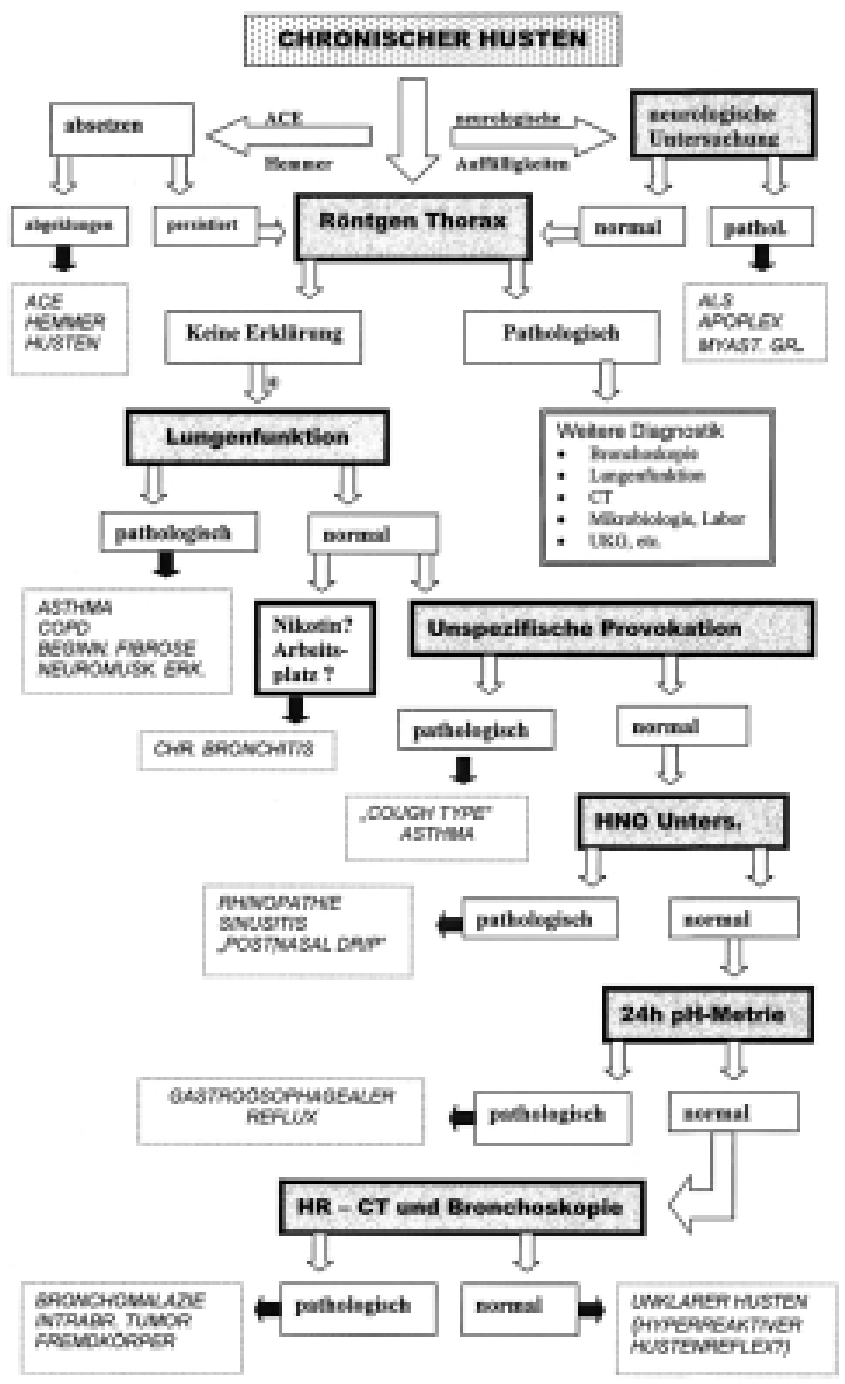

Abb. 2 Flußdiagramm chronischer Husten

*Anmerkung: Bei voluminösem Auswurf kann es zweckmäßig sein, anstelle der Lungenfunktion eine hochauflösende Computertomographie des Thorax zu veranlassen.

Husten unter ACE-Hemmer-Medikation nimmt eine Sonderstellung ein: Hier kann ein Auslaßversuch ohne weitere Diagnostik indiziert sein. Falls der Husten innerhalb einer Woche verschwindet, kann ggf. auf die weitere Diagnostik verzichtet werden. Realistisch erscheint die Annahme, daß etwa $10 \%$ aller Frauen und $5 \%$ der Männer unter ACEHemmer-Medikation husten, in der Literatur werden Zahlen zwischen $0,2 \%$ und $33 \%$ genannt [9].

\section{Chronisch persistierender Husten (CPH)}

Im Rahmen der diagnostischen Abklärung eines Patienten mit chronischem Husten erweist sich als wichtigstes weiterführendes Kriterium, ob die Basisdiagnostik bereits richtungweisend ist (z.B. Bronchialkarzinom) oder aber sie läßt die Ursache weiter unklar: Der chronische Husten tritt dann nicht als Symptom einer leicht definierbaren Erkrankung, sondern sozusagen als eigenständiges Krankheitsbild in Erscheinung (Tab. 4). Für einen solchen chronischen Husten ohne evidente
Tab. 4 Klassifizierung des chronischen Hustens je nach Ergebnis der Thoraxaufnahme und der Spirographie (der Basisdiagnostik)

Ursache evident

Ursache nicht evident. $\mathrm{CPH}$

(chronisch persistierender Husten)

\begin{tabular}{ll}
\hline Asthma oder COPD & obere Atemwege \\
Bronchialkarzinom & Husten als Asthmaäquivalent \\
Tbc & gastroösophagealer Reflux \\
Lungenparenchymerkrankungen & chronische, nicht obstr. Bronchitis \\
Linksherzinsuffizienz & ACE-Hemmer-Einnahme \\
etc. & seltene Ursachen
\end{tabular}

Ursache schlugen wir in Anlehnung an die angelsächsische Literatur den Begriff „chronisch persistierender Husten“ $(\mathrm{CPH})$ vor $[10]$.

CPH wurde definiert als über 8 Wochen anhaltender Husten (produktiv oder trocken), dessen Ursache bei der klinischen Untersuchung sowie Thorax-Röntgenaufnahme und Lungenfunktionsprüfung nicht ersichtlich ist. Irwin et al. schlugen 1977 vor, die Ursache eines solchen CPH entlang der bekannten anatomischen Lokalisation der Hustenrezeptoren zu suchen [7]. Auf der Grundlage dieses anatomisch-diagnostischen Protokolls wurden mehrere Studien in den USA, im Vereinigten Königreich und in Deutschland durchgeführt und Patientenkollektive (43 bis 329 Patienten mit CPH) ausgewertet $[4,5,11,12,13,15,19]$. Als Ursache für den CPH fanden sich, abgesehen von Einzelfällen, in allen Studien - mit etwas unterschiedlicher Häufigkeit - folgende Erkrankungen, die es abzuklären und $\mathrm{zu}$ behandeln gilt, um den Husten $\mathrm{zu}$ kurieren:

- Erkrankungen im Bereich der oberen Atemwege wie Sinusitis, Pharyngitis, Rhinopathie meist im Sinne von NENAR (non-eosinophil, non-allergic rhinopathia) oder NARES (non-allergic rhinopathia eosinophilia syndrome), oft mit sog. „postnasal drip“ (Schleimstraßen im Rachen, Irritationsgefühl im Hals, Globus, Hustenreiz, Räusperzwang). Diese alläglichen Erkrankungen führen nicht bei jedem Patienten zu CPH, viele CPH-Patienten husten aber so lange weiter, bis ihre beispielsweise chronische Rhinopathie nicht saniert wird. Für den Allgemeinarzt (Kinderarzt, Internisten oder Pneumologen) ist diese Diagnose eine besondere Herausforderung, da er in der Regel die entsprechenden Untersuchungstechniken nicht beherrscht und den Patienten zum Hals-Nasen-Ohrenarzt überweisen muß. Polypen im Bereich der Sinus ethmoidalis und sphenoidalis sind häufig nur computertomographisch zu diagnostizieren.

- CPH kann auch Ausdruck eines rudimentären Asthma bronchiale sein. Diese Patienten weisen neben trockenem Husten eine bronchiale Hyperreagibilität auf. Die beiden anderen Symptome des Asthmas: Atemnot sowie Pfeifen und Brummen, d.h. die Bronchialobstruktion fehlt, daher läßt sich dieser „Husten als Asthmaäquivalent“ („cough type asthma“, „cough variant asthma“) spirographisch nicht diagnostizieren. Die Verdachtsdiagnose wird auf Grund der Klinik bei Nachweis der bronchialen Hyperreagibilität im unspezifischen inhalativen Provokationstest gestellt und durch Ansprechen auf die antiasthmatische Therapie bestätigt. 
- Gastroösophagealer Reflux (GÖR), mit oder ohne Sodbrennen [3], kann ebenfalls CPH verursachen. GÖR läßt sich nur mittels 24-Stunden-pH-Metrie diagnostizieren, da er auch ohne Ösophagitis vorkommen kann. Eine pathologische Säureexpositionszeit im unteren Ösophagus bei $\mathrm{CPH}$ ist stets ein Hinweis auf den kausalen Zusammenhang; ohne pathologische Säureexpositionszeit bei häufigen mit $\mathrm{Hu}-$ sten zeitlich assoziierten Refluxepisoden ist auch an die Kausalkette Reflux $\rightarrow$ Husten zu denken $[6,8]$. Die pHmetrisch gestellte Diagnose eines refluxbedingten Hustens bedarf der Bestätigung durch eine erfolgreiche Antirefluxtherapie mit Protonenpumpenhemmern. Eine ex juvantibus-Behandlung ohne pH-Metrie ist nicht unproblematisch, da die durchschnittliche Ansprechzeit auf die Therapie 53 Tage (7-90 Tage) beträgt [16].

- Die chronische Bronchitis wird nach der gängigen WHODefinition als Husten (und Auswurf an den meisten Tagen in mindestens 3 Monaten in 2 aufeinanderfolgenden Jahren) definiert. Die nichtobstruktive Variante geht mit normaler Lungenfunktion einher und gehört in das Spektrum des CPH. Sie kann aber nur dann als Ursache für den $\mathrm{CPH}$ akzeptiert werden, falls der chronischen Bronchitis eine identifizierbare Ursache (Rauchen, arbeitsplatzbezogene Schadstoffbelastung) zugrundeliegt und weitere Erkrankungen, die Husten hervorrufen können, fehlen. In allen CPH-Studien (s. oben) lag der Anteil der Raucher, (falls nicht von vornherein ausgeschlossen [12]) auffallend niedrig. Offensichtlich verdrängen die Raucher den obligaten „Raucherhusten“ und suchen damit den Arzt gar nicht erst auf.

- Bronchiektasen können auf der Übersichtsaufnahme des Thorax leicht übersehen werden und so in die Kategorie des $\mathrm{CPH}$ fallen. Sie verursachen meist Husten mit voluminösem Auswurf (mindestens $30 \mathrm{ml}$, entsprechend 2 Eßlöffel voll pro 24 Stunden). Allerdings sind Diagnostik und das mögliche Diagnosespektrum des produktiven Hustens nicht grundsätzlich anders als des sogenannten trockenen Reizhustens [15]. Bei voluminösem Auswurf und nichtdiagnostischer Röntgen-Thoraxaufnahme führt abweichend vom Flußdiagramm in Abb. 2 anstelle der Lungenfunktion eine hochauflösende Computertomographie des Thorax auf dem rationellsten Weg zur Diagnose. Diese Stelle ist in Abb. 2 mit einem * gekennzeichnet.

- In zwei [5] bis ca. 20 Prozent [11,12] aller Patienten mit $\mathrm{CPH}$ bleibt - ungeachtet einer extensiven Diagnostik - die Ursache des Hustens unklar. Nach unserer Erfahrung läßt die Mehrzahl dieser Patientinnen - Verhältnis Frauen:Männer $=2: 1$ - eine erhöhte Sensitivität des Hustenreflexes gegenüber Capsaicin (Husten bereits bei Inhalation einer Konzentration von $1-10 \mu \mathrm{mol} / \mathrm{ml}$ ) erkennen, welche den schon auf unterschwellige Reize einsetzenden Husten erklären könnte.

- Im Gegensatz hierzu scheint der psychogene Husten - ohne Erniedrigung der Capsaicin-Reizschwelle - bei Erwachsenen extrem selten (bei Kindern häufiger) zu sein. Allerdings ergibt die Bestimmung der Capsaicin-Reizschwelle gerade beim psychogenen Husten schlecht reproduzierbare Ergebnisse, weshalb die Methode bislang keine praktische Bedeutung in der klinischen Routine erlangen konnte.

- In seltenen Fällen verursacht Bronchomalazie mit taktilem Kontakt zwischen Vorderwand und Hinterwand beispielsweise des Intermediärbronchus bereits bei Spontanatmung oder bei leicht forcierter Exspiration (Funktionsbronchoskopie) therapieresistenten CPH $[10,19]$.

- Patienten mit Bulbärparalyse oder Pseudobulbärparalyse im Rahmen verschiedener neurologischer Erkrankungen, auch M. Parkinson, leiden oft an CPH, dessen Ursache in der Schluckstörung liegt: Entweder kommt es zu häufigen Mikroaspirationen, oder verursacht der Speichelstau im Hals in Verbindung mit dem gestörten Schluckakt Husten.

Dem CPH liegen oft sehr häufige, banale Befunde zugrunde, welche auch ohne Husten vorkommen. Daher reicht beispielsweise der methodisch einwandfreie Nachweis einer bronchialen Hyperreagibilität keinesfalls aus, um damit den Husten endgültig zu erklären: Nur wenn der Patient auf die entsprechende Therapie (hier z.B. auf inhalative Steroide oder Nedocromil) anspricht, kann die Diagnose endgültig gestellt werden. Viele CPH-Patienten leiden gleichzeitig an zwei oder gar drei Erkrankungen (z.B. Husten als Asthmaäquivalent und Rhinopathie). Jede Erkrankung muß für sich behandelt werden, um den Husten zu bessern. Das Flußdiagramm, wie in Abb. 2 dargestellt, wird dem multikausalen CPH unter Umständen nicht gerecht. Bei Therapieversagen sollte stets an multiple Ursachen gedacht werden.

\section{Wie kann man diagnostische Maßnahmen einsparen?}

Durch Einhaltung der Stufendiagnostik können oft Untersuchungen ohne Nachteil für den Patienten eingespart werden.

- Einem IgE vermittelten (atopisch-)allergischen Husten kann nur entweder ein („cough variant“) Asthma oder eine allergische Rhinopathie zugrunde liegen. Wenn Asthma und Rhinopathie auszuschließen sind, ist die gesamte allergologische Diagnostik in Hinblick auf die Abklärung des Hustens überflüssig.

- Serologische Untersuchungen auf Viren und Bakterien (z. B. Mycoplasma pneumoniae- und Bordatella pertussis-Serologie) können selten einen $\mathrm{CPH}$ erklären. Sie sollten in unklaren Fällen ohne konkreten Verdacht (z.B. Kontaktperson) nicht am Anfang, sondern am Ende der Stufendiagnostik stehen.

- Falls die Basisdiagnostik (Tab.2) keinen Hinweis für die mögliche Ursache des Hustens liefert, liegt die Wahrscheinlichkeit, daß der Husten bronchoskopisch abgeklärt werden kann, bei unter 5\% [11,14]. Es besteht dabei Konsens, dass jeder Patient mit unklarem chronischen Husten bronchoskopiert werden muß, nur steht die Bronchoskopie unter dem Gesichtspunkt der rationalen und rationellen Diagnostik nicht am Anfang, sondern am Ende der Diagnostik, wie im Flussdiagramm (Abb.2) dargestellt. Wir sehen häufig Patienten, die zur Bronchoskopie überwiesen werden, noch bevor die unspezifische bronchiale Provokationstestung durchgeführt wurde.

- Patienten mit massiv beeinträchtigter Nasenatmung als Ausdruck einer Rhinopathie sollten zunächst dem HalsNasen-Ohrenarzt vorgestellt und nicht etwa gleich bronchoskopiert werden.

- Die Ösophagoskopie und die Röntgenuntersuchung des Ösophagus sind nicht geeignet, einen GÖR als Ursache des Hustens auszuschließen und gar die Assoziation zwischen Refluxepisoden und Husten zu demonstrieren. Zur Abklärung des Hustens sollte daher erst die 24-Stunden-pHMetrie durchgeführt werden. Bei pH-metrisch bestätigtem Reflux mit assoziiertem Husten gilt die Gastroskopie als 
weiterführende, erforderliche Diagnostik - allerdings nur dann, wenn Reflux vorliegt (und auch dann nicht mehr sensu stricto - zur Abklärung des Hustens).

Die Rationalität bei der Abklärung des Hustens wird am häufigsten dadurch verletzt, daß in vielen Fällen nicht die am ehesten erfolgversprechende Diagnostik (die unspezifische Provokation, die hals-nasen-ohrenärztliche Untersuchung oder die 24-h-pH-Metrie), sondern die am leichtesten verfügbaren Untersuchungen durchgeführt werden.

\section{Schlußfolgerung}

Husten ist das häufigste Symptom bei pneumologischen Patienten. Eine rationale wie auch rationelle Abklärung des Hustens ist unter dem Aspekt seiner Häufigkeit sehr wichtig. Der überwiegenden Mehrzahl der Patienten wird man aus diagnostischer Sicht gerecht, wenn nur einige wenige Punkte in dem diagnostischen Stufenschema konsequent berücksichtigt werden:

1. Die häufigste Ursache des Hustens überhaupt ist die akute Bronchitis, ggf. im Rahmen eines grippalen Infektes, die meistens erst nach drei bis vier Wochen - abklingt und in der Regel außer Anamnese und körperlicher Untersuchung keine weiteren diagnostischen Maßnahmen erfordert.

2. Die breit verfügbare und kostengünstige Basisdiagnostik (Tab. 2) reicht in vielen Fällen aus, den ersten diagnostischen Verdacht in Hinblick auf die Ursache des Symptoms Husten auszusprechen und eine gezielte Diagnostik der Grundkrankheit einzuleiten.

3. Sollte die Ursache des Hustens nach der Basisdiagnostik unklar bleiben (z.B. CPH), ist ein gezieltes diagnostisches Vorgehen in Hinblick auf die Wahrscheinlichkeit möglicher Diagnosen, auf den positiven prädiktiven Wert, Kosten und Invasivität einzelner diagnostischen Methoden angezeigt. Die Abb. 1 und $\mathbf{2}$ zeigen je ein Flußdiagramm für den sinnvollen Einsatz der Stufendiagnostik bei akutem bzw. chronischem Husten.

4. Falls es zunächst nicht gelingt, den Husten nach vorausgegangener Diagnostik durch eine gezielte „kausale“ Therapie zu bessern, ist an Husten multikausaler Ursache zu denken, weitere diagnostische Maßnahmen sind dann indiziert.

\section{Literatur}

${ }^{1}$ Office Visits to Internists. The National Ambulatory Medical Care Survey 1975. Washington D.C: US DHEW Publication PHS, 1978. $79-1787$

${ }^{2}$ Holmes WF, Macfarlane JT, Macfarlane RM, Lewis S. The Influence Of Antibiotics And Other factors on reconsultation for acute lower respiratory tract illness in primary care. $\mathrm{Br} \mathrm{J}$ Gen Pract 1997; 47: 815 - 818

${ }^{3}$ Ing AJ, Ngu MC, Breslin AB. Chronic Persistent Cough And Gastro-oesophageal Reflux. Thorax 1991; 46: 479 - 483

${ }^{4}$ Irwin RS, Corrao WM, Pratter MR. Chronic Persistent Cough In The Adult: the spectrum and frequency of causes and successful outcome of specific therapy. Am Rev Respir Dis 1981; 123: 413 417

${ }^{5}$ Irwin RS, Curley FJ, French CL. Chronic Cough. The Spectrum And Frequency of causes, key components of the diagnostic evaluation, and outcome of specific therapy. Am Rev Respir Dis 1990; 141: $640-647$
${ }^{6}$ Irwin RS, French CL, Curley FJ, Zawacki JK, Bennett FM. Chronic Cough Due To Gastroesophageal Reflux. Clinical, diagnostic, and pathogenetic aspects. Chest 1993; 104: $1511-1517$

${ }^{7}$ Irwin RS, Rosen MJ, Braman SS. Cough. A comprehensive review. Arch Intern Med 1977; 137: 1186 - 1191

${ }^{8}$ Irwin RS, Zawacki JK, Curley FJ, French CL, Hoffman PJ. Chronic Cough As The Sole Presenting manifestation of gastroesophageal reflux. Am Rev Respir Dis 1989; 140: 1294 - 1300

${ }^{9}$ Israili ZH, Hall WD. Cough And Angioneurotic Edema Associated With angiotensin-converting enzyme inhibitor therapy. A review of the literature and pathophysiology. Ann Intern Med 1992; 117: $234-242$

${ }^{10}$ Kardos P. Chronisch persistierender Husten (CPH). Pneumologie 1995; 49: $2-13$

${ }^{11}$ Kardos P, Gebhardt T. Chronisch persistierender Husten (CPH) in der Praxis: Diagnostik und Therapie bei 329 Patienten in 2 Jahren. Pneumologie 1996; 50: 437 - 441

12 McGarvey LA, Heaney LG, Lawson JT et al. Evaluation and outcome of patients with chronic non-productive cough using a comprehensive diagnostic protocol Thorax 1998; 53: 738 - 743

${ }^{13}$ Poe RH, Harder RV, Israel RH, Kallay MC. Chronic Persistent Cough. Experience In Diagnosis and outcome using an anatomic diagnostic protocol. Chest 1989; 95: 723 - 728

${ }^{14}$ Poe RH, Israel RH, Utell MJ, Hall WJ. Chronic Cough: Bronchoscopy Or Pulmonary Function testing?. Am Rev Respir Dis 1982; 126: $160-162$

${ }^{15}$ Smyrnios NA, Irwin RS, Curley FJ. Chronic Cough With A History Of excessive sputum production. The spectrum and frequency of causes, key components of the diagnostic evaluation, and outcome of specific therapy. Chest 1995; 108: 991 - 997

${ }^{16}$ Vaezi MF, Richter JE. Twenty-four-hour Ambulatory Esophageal $\mathrm{PH}$ Monitoring In the diagnosis of acid reflux-related chronic cough. South Med J 1997; 90: 305 - 311

${ }^{17}$ Vozehova S. [Subjective Health Problems In Persons 60-64 years of age and over 65] Subjektivni zdravotni potize osob ve veku 60-64 let a nad 65 let. Cesk Zdrav 1989; 37: 473 - 477

${ }^{18}$ American College of Chest Physicians. Managing Cough as a Defense Mechanism and as a Symptom. Clinical Consensus Statement Chest 1998; 114: 133S - 181S

${ }^{19}$ Palombini BC, Villanova CAC, Araujo E et al.. A Pathogenic Triad in Chronic Chough. Chest 1999; 116: 279 - 284

Dr. med. Peter Kardos

Scheffelstraße 33

60318 Frankfurt am Main

E-mail: Peter.Kardos@Frankfurt.netsurf.de 